\title{
Fuzzy Differential Inclusions in Atmospheric and Medical Cybernetics
}

\author{
Kausik Kumar Majumdar and Dwijesh Dutta Majumder
}

\begin{abstract}
Uncertainty management in dynamical systems is receiving attention in artificial intelligence, particularly in the fields of qualitative and model based reasoning. Fuzzy dynamical systems occupy a very important position in the class of uncertain systems. It is well established that the fuzzy dynamical systems represented by a set of fuzzy differential inclusions (FDI) are very convenient tools for modeling and simulation of various uncertain systems. In this paper, we discuss about the mathematical modeling of two very complex natural phenomena by means of FDIs. One of them belongs to the atmospheric cybernetics (the term has been used in a broad sense) of the genesis of a cyclonic storm (cyclogenesis), and the other belongs to the bio-medical cybernetics of the evolution of tumor in a human body. Since a discussion of the former already appears in a previous paper by the first author, here, we present very briefly a theoretical formalism of cyclone formation. On the other hand, we treat the latter system more elaborately. We solve the FDIs with the help of an algorithm developed in this paper to numerically simulate the mathematical models. From the simulation results thus obtained, we have drawn a number of interesting conclusions, which have been verified, and this vindicates the validity of our models.
\end{abstract}

Index Terms-Carcinogenesis, cybernetics, cyclogenesis, fuzzy differential inclusions, fuzzy dynamical systems.

\section{INTRODUCTION}

C YBERNETICS means the control and communication in a real machine-electronic, mechanical, neural, or economic [1]. In this paper, we add two more to this list, namely, an atmospheric machine (the genesis of an intense tropical storm over the sea) and the physiological machine (the evolution of a tumor in a human body). Classically, cybernetics was concerned only about determinate machines [1], [3]. That is the machine whose state at any time can be uniquely predicted if the initial state is known. During the 1960s, Bertalanffy developed general systems theory (GST) that included biological systems in general [38]. Majumder, in the late 1970s, developed a mathematical formalism of cybernetics and GST as a unitary science [24], but following the footsteps of the quantum mechanics, the ubiquity of uncertainty in the real world is very well accepted these days. With this came the notion of uncertain dynamical systems, where neither the initial states nor the equations representing the system can be precisely known. Moreover, the nature of uncertainty involved may not always be statistical. Chang and Zadeh introduced the notion of fuzzy dynamical systems (FDSs)

Manuscript received May 31, 2002; revised October 9, 2002 and February 20, 2003. This paper was recommended by Associate Editor M. Zhou

The authors are with the Electronics and Communications Sciences Unit, Indian Statistical Institute, Calcutta, 700108, India.

Digital Object Identifier 10.1109/TSMCB.2003.817105
[4], whose most prominent subclass is the class of fuzzy controllers [58], [59]. In an atmospheric dynamical system or in a physiological dynamical system, which is the theoretical model of an atmospheric or physiological dynamic machine, the role of uncertainty is much greater compared to classical physics, mechanics, or engineering. Right here, the unitary formalism of cybernetics and GST play the role of a great integrator of ideas emerging from diverse fields of physics, biology, and engineering. This enables the scientists to understand multidisciplinary complex problems. In this paper, our aim is to discuss the methodologies to model a phenomenon from atmospheric physics and another from human biology.

Historically, the mathematical theory of FDS was almost simultaneously but independently introduced by Kloeden [5] and de Glas [6]. The fuzzy differentiable dynamical system (FDDS) was invented by Kaleva [7] and Seikkala [8] in the form of time dependent fuzzy differential equations (FDEs). The addition of two fuzzy numbers produces another fuzzy number whose diameter (as the diameter of a fuzzy set) is in general greater than the diameter of each of the constituent fuzzy numbers. This implies that the diameter of the fuzzy point obtained as the solution of a classical FDE at a particular time $t$ is in general an increasing function in $t$, with the property that as $t$ tends to infinity, the diameter of the corresponding fuzzy point also generally becomes infinite. This renders the FDEs unsuitable for modeling and simulation. To overcome this difficulty, first, Aubin [36] and then Hullermeier [2] introduced the notion of the FDI relation. In [12], Hullermeier has devised an algorithm to numerically solve general FDIs. Diamond has extended some of the classical dynamical system theoretic notions to the fuzzy dynamical systems in general and to the systems represented by FDIs in particular [39], [40]. FDIs have turned out to be great tools to tackle complexity due to uncertainty in an FDS [9], [10]. Differential inclusions and fuzzy differential inclusions have already been applied in population dynamics [35], [41]. In this paper, we discuss the role of FDIs in modeling and simulation of highly uncertain (and, therefore, very complex) dynamics of atmospheric and biological cybernetics.

In Section II, we will present a brief introduction to FDIs and present an algorithm (christened as the Crystalline Algorithm) for the numerical simulation of a system represented by a set of one-dimensional (1-D) FDIs. In this section, as an example, we have described a system represented by a set of 1-D FDIs and given the numerical simulation of the system according to the Crystalline Algorithm. In Section III, we have discussed the first ever application of FDIs in atmospheric science [9], [37]. Here, we have briefly reviewed the work presented in [9] and [37]. In Section IV, we have viewed the evolution of tumor in a human 
body from a general system theoretic point of view, as done in [24], [25], [29], and [31]. We have presented an FDI model of the phenomenon and have suggested a therapeutic remedy of malignancy along with its major limitations based on this model. Section V contains the summary and conclusion of this paper.

\section{FUZZY DifFERENTIAL INCLUSION}

An FDI relation, as introduced in [2] and [36], is written as

$$
\mathrm{x}^{\prime}(\mathrm{t}) \in[\mathrm{f}(\mathrm{t}, \mathrm{x}(\mathrm{t}))]^{\alpha}, \quad \mathrm{x}(0) \in\left[\mathrm{x}_{0}\right]^{\alpha} .
$$

$\mathrm{f}(\mathrm{t}, \mathrm{x}(\mathrm{t}))$ is a fuzzy valued continuous function defined on $\mathrm{E}^{\mathrm{n}}$, which is the collection of all normal, upper semi-continuous, convex, compact supported fuzzy subsets of $\mathrm{R}^{\mathrm{n}}$ [2], [7], [8], [39], and [40]. $\mathrm{x}(\mathrm{t})$ is a variable crisp trajectory (a continuous curve starting at one point and ending up at another) varying over an entire fuzzy set of crisp trajectories. $\mathrm{x}_{0}$ is the fuzzy set to which all possible initial values of $\mathrm{x}(\mathrm{t})$ belong. $0<\alpha \leq 1$. The fuzzy set of crisp trajectories is the fuzzy solution of (2.1). $\mathrm{x}^{\prime}(\mathrm{t})$ is the ordinary crisp derivative of $\mathrm{x}(\mathrm{t})$ with respect to $t$ (time).

To understand the FDI relation (2.1), let us first consider the ordinary crisp differential equation

$$
x^{\prime}(t)=f(t, x(t)), \quad x(0)=x_{0} .
$$

Here, everything is very specific and absolutely without any chance of uncertainty in determining the values of the variables, constants, and the form of the equation(s), but in reality, it may so happen (and it does indeed happen in many cases) that we may not be able to determine the value of $\mathrm{x}(0)$ and the values of parameters of $f(t, x(t))$ very precisely. Each measurement may involve some fluctuation due to uncertainty. The more you want to be accurate in your measurements, the more complex your job will be. So to keep the complexity within some manageable limit, we actually allow some uncertainty to remain for ever with our measurement [11]. In the process, $x_{0}$ becomes a fuzzy set of possible values of $x(0)$, and $f(t, x(t))$ becomes a fuzzy set of crisp functions. Any specific form in the fuzzy set of crisp functions $\mathrm{f}(\mathrm{t}, \mathrm{x}(\mathrm{t}))$ and any specific value of $\mathrm{x}(0)$ in $\mathrm{x}_{0}$ may satisfy (2.2) up to a certain possibility measure or membership value. So we allow $\mathrm{f}(\mathrm{t}, \mathrm{x}(\mathrm{t}))$ and $\mathrm{x}_{0}$ in (2.2) to become fuzzy sets, and consequently, the equal signs in (2.2) become meaningless. All "=" are replaced by " $\in$ " (inclusion), and (2.2) takes the form of (2.1). Moreover, we may exercise the freedom of restricting our choice to certain $\alpha$-level subsets of the fuzzy sets in order to ignore solutions that represent the system behavior (modeled by (2.1)) with a degree of possibility less than $\alpha$, where $0<\alpha \leq 1$. Obviously, the best system behavior is represented by the solutions of (2.1) for $\alpha=1$.

\section{A. Algorithm}

Numerical simulation of FDIs has been discussed in detail in [2] and [12]. In [12], an algorithm to numerically solve general FDIs has been described. In this paper, we have found an easier algorithm to solve 1-D FDIs, which cannot be extended to the multidimensional cases. In 1-D cases, our algorithm is easier to implement and less computation intensive compared to that in [12].

\section{Crystalline Algorithm [Solution of FDIs]:}

1) Fix $\alpha \in[0,1]$.

2) Calculate $[\mathrm{f}(\mathrm{t}, \mathrm{x}(\mathrm{t}))]^{\alpha}$ by Zadeh's extension principle [13] or otherwise as the case may be (the $\alpha$-level set $[\mathrm{f}(\mathrm{t}, \mathrm{x}(\mathrm{t}))]^{\alpha}$ is an $n$-tuple of $\alpha$-cuts of fuzzy numbers for some $n$ ).

3) Solve (directly or numerically) the ordinary crisp DE $\mathrm{x}^{\prime}(\mathrm{t})=\mathrm{f}(\mathrm{t}, \mathrm{x}(\mathrm{t})), \mathrm{x}(0)=\mathrm{x}_{0}$ only for the boundary values of the $\alpha$-level set of step 2 .

4) The region of $R^{2}$ enclosed by the graphs of the solutions obtained in step 3 , possibly along with the coordinate axes, is the $\alpha$-level set of the solution of the FDI (2.1) for the fixed $\alpha$ of step 1 .

\section{B. Example}

Find the solution representing the best possible behavior of the system given by

$$
\begin{aligned}
\mathrm{x}^{\prime \prime}(\mathrm{t}) & \in[\mathrm{kx}]^{\alpha} \\
\mathrm{k} & \in[\mathrm{K}]^{\alpha} \\
\mathrm{x}^{\prime}(0) & \in[\mathrm{L}]^{\alpha} \quad \text { for } \mathrm{x}(\mathrm{t}) \in[\mathrm{M}]^{\alpha} \\
\mathrm{x}(0) & \in\left[\mathrm{x}_{0}\right]^{\alpha}
\end{aligned}
$$

where $\mathrm{x}^{\prime \prime}(\mathrm{t})=\mathrm{dx}^{\prime}(\mathrm{t}) / \mathrm{dt}$. $\mathrm{K}$ is a trapezoidal fuzzy number $\langle 0.4,0.6,1,1.2\rangle \cdot \mathrm{x}_{0}$ is a triangular fuzzy number $\left.\langle-\delta, 0, \delta\rangle, \delta\right\rangle$ 0 . $\mathrm{L}$ is a triangular fuzzy number $\langle-\lambda, 0, \lambda\rangle, \lambda>0$. M is a triangular fuzzy number $\langle 1-\lambda, 1,1+\lambda\rangle, \lambda>0.0 \leq \alpha \leq 1$. We also show the simulated solution (state) space of the system.

1) Solution: Notice that we are to determine the solution representing the best system behavior only. In that case, $\alpha=1$. So $\mathrm{k} \in[\mathrm{K}]^{1}=[0.6,1] \mathrm{x}(\mathrm{t})$ is a crisp variable, but $\mathrm{x}(0)$ can take any crisp value from a fuzzy number. This contributes to the "fuzziness" of $\mathrm{x}(\mathrm{t})$ (there may as well be other factors to contribute in this direction), that is, $\mathrm{x}(\mathrm{t})$ becomes a crisp member of a fuzzy set of crisp functions. So in search of a seed solution, it suffices to take

$$
\mathrm{x}^{\prime \prime}(\mathrm{t})=\mathrm{kx}
$$

where $\mathrm{k} \in[0.6,1]$, and $\mathrm{x}$ is an ordinary crisp variable. We shall proceed by solving (2.7) just like an ordinary crisp differential equation. Multiplying both sides of (2.7) by $2 \mathrm{x}^{\prime}(\mathrm{t})$ and then integrating, we get

$$
\left(\mathrm{x}^{\prime}\right)^{2}=\mathrm{kx}^{2}+\mathrm{C}
$$

where $\mathrm{C}$ is an integration constant. From (2.5), we get that $\mathrm{x}^{\prime}(0) \in[\mathrm{L}]^{\alpha}$ for $\mathrm{x}(\mathrm{t}) \in[\mathrm{M}]^{\alpha}$, which means $\mathrm{x}^{\prime}(0) \in[\mathrm{L}]^{1}$ for $\mathrm{x}(\mathrm{t}) \in[\mathrm{M}]^{1}$. Since $\mathrm{L}$ and $\mathrm{M}$, are triangular fuzzy numbers of $\langle-\lambda, 0, \lambda\rangle$ and $\langle 1-\lambda, 1,1+\lambda\rangle$, respectively, we have $\mathrm{x}^{\prime}(0)=0$ for $\mathrm{x}(\mathrm{t})=1$. Hence, from (2.8), we get $\mathrm{C}=-\mathrm{k}$, or

$$
\mathrm{x}^{\prime}(\mathrm{t})=\sqrt{ } \mathrm{k}\left(\mathrm{x}^{2}-1\right)^{1 / 2} .
$$

Solving (2.9), we get

$$
\sin ^{-1} x(t)=\sqrt{ } k t+D
$$


where $\mathrm{D}$ is an integration constant, and we are considering the positive square root of $\mathrm{k}$ only. From (2.6), we get $\mathrm{x}(0) \in\left[\mathrm{x}_{0}\right]^{0}$, but we are interested only in $\mathrm{x}(0) \in\left[\mathrm{x}_{0}\right]^{1}$ or $\mathrm{x}(0)=0$. So

$$
\mathrm{x}(\mathrm{t})=\sin (\sqrt{ } \mathrm{kt})
$$

where $\mathrm{k} \in[0.6,1]$. Equation (2.11) is the seed solution of (2.3)(2.6) for any $\mathrm{k} \in[0.6,1]$. To determine the fuzzy flow corresponding to the best possible behavior of the system represented by (2.3)-(2.6) in the phase space, we shall have to determine the boundary of the lighter fuzzy flow in $\mathrm{R}^{2}$. The boundary is represented by the graphs of $\mathrm{x}(\mathrm{t})=\sin (0.7746 \mathrm{t})$ and $\mathrm{x}(\mathrm{t})=\sin (\mathrm{t})$.

The general situation when $\alpha<1$ has been indicated by the entire fuzzy flow colored by darker fuzzy flow in Fig. 1 .

\section{FDI Modeling of CyClogenesis: A BRIEF SuRveY}

With earthquakes and volcanic eruptions, tropical cyclones (known as hurricanes in the United States) rank among the most catastrophic natural disasters on earth. Because of their devastating power, the study of cyclones has always held much scientific interest. Decades of intensive and extensive study has resulted in some understanding of the physics behind such storms. A standard way of research is making mathematical models of the storm and then trying to make the models more and more realistic in order to study and predict various aspects of storms as accurately as possible. A remarkable success in this direction was achieved by Ooyama in 1969 [17]. Numerous models of cyclones have been proposed, and every now and then, a new mathematical model of a tropical storm appears in the atmospheric science journals. Different models focus on different aspects of the storms. Each model is represented by a set of equations, some of which are partial differential equations. Some of these equations are time dependent. The equations typically govern the fluid dynamic and thermodynamic aspects of a storm. A storm is modeled as a cylindrical vortex of 20 to $200 \mathrm{~km}$ in diameter spanning from the sea surface to up to $15 \mathrm{~km}$ above like a big trough. In a mature intense storm, this vortex may revolve around its axis at an angular speed of about 10 revolutions/hr. The energy of this huge vortex is supplied by the enormous heat reserve of ocean water at the tropical region (where sun rays fall more or less normally). This heat induces a convective current into the surrounding atmosphere, which in turn keeps the cyclonic vortex rotating by supplying the required angular momentum. The detail of these mechanisms may be seen in [15], [17], [18], and [20]. Due to variation in air pressure this huge cyclonic vortex, which most often forms on the seas in the tropics, always has a propensity to drag toward the land. As long as it remains on the sea (usually for five to six days), it intensifies by consuming heat energy from the warm tropical sea water. The more it intensifies, the greater the rotational speed of the vortex. Its real devastation begins on reaching the coast. If at the time of hitting the coast the vortex is revolving around its axis at a rate of 8 revolutions $/ \mathrm{hr}$ and the radius is $20 \mathrm{~km}$, then the maximum wind speed of the cyclone will be $8 \times 20=160 \mathrm{~km} / \mathrm{h}$. An early forecast of such devastation is of utmost importance and, hence, the need for reliable models.

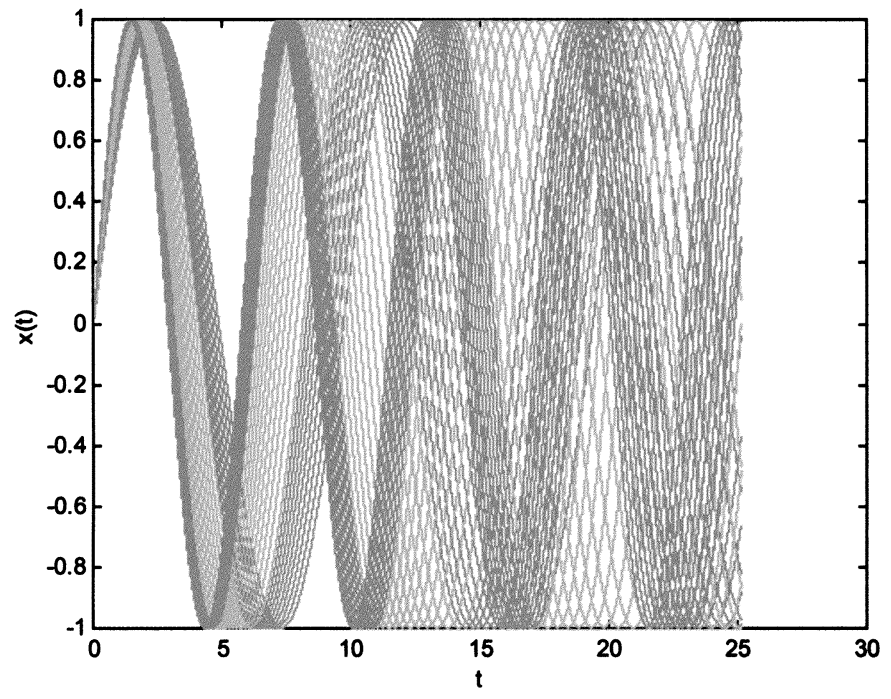

Fig. 1. Fuzzy state space of the system represented by (2.3)-(2.6). The lighter flow is representing the best system behavior has been considered. The darker flow represents the system behavior for $\alpha<1$ (assuming $\delta \rightarrow 0$ and $\lambda \rightarrow 0$ ).

In a mathematical model represented by a set of equations, the variables and parameters are all measurable quantities. Unfortunately, the precise measurement of the atmospheric quantities is very difficult. Almost always, a lot of uncertainties remain involved in any such measurement. Therefore, a precise mathematical formulation of a system like a cyclone may be theoretically beautiful, but practically, it is unrealistic. Here, we have the fuzzy dynamical system. In [9], one of the present authors has demonstrated the power of FDIs in modeling an atmospheric system like the generation of cyclones. To the best of our knowledge, this is the first ever attempt to apply fuzzy set theory-based techniques to atmospheric science. Here, we present a brief review of that model.

It is well known that favorable climatic and geographical conditions for the formation of a tropical storm of sufficient intensity prevail over large part of the globe during the storm seasons, yet the actual occurrence of a strong tropical storm of hurricane strength (wind speed $90 \mathrm{~km} / \mathrm{h}$ or more) is a relatively rare phenomenon [15], [16]. It is believed that along with other favorable conditions, a sufficiently strong initial disturbance is absolutely necessary to generate a storm of hurricane strength. This has also been supported by extensive numerical modeling and simulation [15], but the nature of this initial disturbance is not yet clear to the scientists [15], [18]. One of the present authors has tried to shed some light on this aspect by proposing a mathematical model of this strong initial climatic disturbance, as a precursor for a cyclone, by means of fuzzy differential inclusion relations in [9] and [37], where it has been clearly demonstrated how a logspiral-shaped (the shape of a cyclonic vortex as seen from the satellite and RADAR images [14], [19]) vortex can be created from the collision of two linear wind jets propagating parallel to the ground. One of them is of high intensity (speed $40 \mathrm{~km} / \mathrm{h}$ or more), and the other is very weak (speed about 5 $\mathrm{km} / \mathrm{h}$ ). The first one is much more significant, because it is rare. The second one almost always remains present. Under favorable conditions [9], the created vortex may mature into a severe cyclone. 
In this model, all the uncertain quantities are fuzzy numbers. We have solved the FDI by Crystalline Algorithm, and the simulation gives us the initial spiral shaped cyclonic vortex. Once the initial vortex is created, its transformation into a steady-state cyclone may be modeled in the usual way.

This model has a number of advantages, as for example, it can give a satisfactory explanation of the phenomena where the most intense cyclonic storms occur either during April and May (just before the onset of the monsoon in the Indian sub-continent) or during October and November (just after the withdrawal of the monsoon from the Indian subcontinent). During these times of the year, major changes take place in the wind pattern over the seas surrounding the Indian coasts (Indian Ocean, Bay of Bengal, and Arabian Sea). The wind changes course from land-to-sea to sea-to-land (April-May) and from sea-to-land to land-to-sea (October-November). Naturally, during these periods, the possibility of simultaneous occurrence of one major and one minor disturbances as described above is very high, and hence, there will be the relative high frequency of intense cyclones.

According to this model, the knowledge of the speed of wind, above certain threshold levels and coming from different directions over a region, can be utilized to detect the formation of a cyclone at a very early stage, even earlier than what we usually get today.

\section{EVOLUTION OF TUMOR}

The evolution of a tumor turning malignant (carcinogenesis) is a very complex physiological process that scientists have yet to understand fully. Several attempts are being made to better understand the process from various points of view. Here, we propose to view the entire physiological process from a cybernetic and GST outlook, as suggested in [24], [25], [29], and [31]. In physics, there are a few wonder equations like Schrodinger's equation, Dirac's equation, Yang-Mill's equation, etc., that may not explain everything in their envisaged areas of application. Yet they can explain many things and play a very important role in the understanding of some of the most complicated behaviors of nature. There is an attempt in biology and physiology to develop mathematical tools using cybernetics and general dynamical system theoretic outlook that is similar to the spirit of physics [25], [29], [31], [35], [43]. Systems theoretic pioneer Bertalanffy initiated this approach as a mathematical biologist in 1968 [38]. His equation is used for study of growth in animals and tumors. Tsetlin had also taken a similar approach in modeling some properties of a muscle and a ganglion [43]. Uncertainties inherent in medical diagnostic systems have long been recognized. Adlassnig attempted to tackle this uncertainty by developing a fuzzy logic-based medical diagnostic expert system [42]. Many more attempts have been made to apply fuzzy set theory based techniques in medical science, but introduction of FDI in modeling a biological system is only a recent trend [41].

Another recent trend in the contemporary dynamical system theoretic studies is the emphasis on fractal sets. The definition of a fractal set as given by Mandelbrot is a complicated one [45].
To us, the most important fact is that, many important dynamical systems have fractal attractors. Usually, a dynamical system which has a fractal attractor often (but not always [33]) turns out to be a chaotic dynamical system (for simplicity, let us assume in this paper that a chaotic dynamical system is one which has at least one positive Liapunov exponent or in other words it has sensitive dependence on initial condition, which means that the system states change exponentially fast, and therefore, it is unpredictable). Studying the fractal attractors is a very standard way to understand such systems [46], [47]. In the last 20 years, this notion has found wide spread application in physical, biological, and social systems. Some works have also been done on fuzzy chaos [48]-[51]. So far, however, they have not found any significant application though an attempt has been made in [51]. Only recently Devaney's comprehensive definition of a (crisp) chaotic dynamical system [52] has been extended to the fuzzy dynamical systems [10], [55]. Fuzzy fractal is an even less explored area. Fuzzy fractals come naturally as attractors of the iterated fuzzy sets systems (IFZS) [60] exactly the same way as the crisp fractals are generated as attractors of contractive iterated function systems [53]. In [54] Cabrelli et al., have treated any gray level image as a fuzzy subset of the euclidean plane. A normalized gray level pixel intensity value at each pixel is taken as the fuzzy membership value of that pixel or point. In [53], IFZSs have been used to generate gray level images. The method used here is a bit complicated. Majumdar has recently introduced some simplifications in this method and proposed a broad based definition for fuzzy fractals, such that, crisp fractals become special cases of fuzzy fractals [55], [60]. Fuzzy chaos and fuzzy fractals have not yet been related as in case of crisp dynamical systems. This is not possible in case of fuzzy differential equation based dynamical systems (due to the same reasons as described in the last paragraph of Subsection C of Section IV). However, FDI relations hold a great promise in this direction. Although we have not used the notions of fuzzy fractals and fuzzy chaos in our modeling of atmospheric and bio-medical cybernetics, fuzzy fractals and fuzzy chaos hold the promise to find useful applications in FDI modeling of complex natural phenomena. A typical outline of exploration for the future researchers should be as follows. Choose a natural phenomenon. Make a model of it in terms of a set of FDI(s). Solve the system of FDI's. Let the solution (phase) space X be bounded. Then, as time $t \rightarrow \infty$ the fuzzy trajectory converges to a subset A of X. A is called the attractor of the underlying dynamical system. Obviously, A is a fuzzy subset of X (fuzzy attractor). In addition, if $A$ is also a fractal subset of $X$, we shall call $A$ a fuzzy fractal [55], [60]. If the dynamical system during evolving on A satisfies the conditions to be a fuzzy chaotic dynamical system as laid down in [10] and [55], then it is a fuzzy chaotic dynamical system with a fuzzy fractal attractor. Many important natural systems will turn out to be of this type. Many important properties of such systems will be found by analyzing the fuzzy fractal attractor.

\section{A. Model}

We present a kinetic model of the onset of tumor that involves the coupling of three principal phenomena [29]: 
1) the transformation of normal into neoplastic cells (tumor cells);

2) the replication of transformed cells;

3) the immunological interaction of the host organizm with transformed cells, i.e., the immunological propensity of the T-lymphocyte (a kind of white blood corpuscle) and some other cells to fight and destroy the unwanted growth of cells in a tumor.

The schematic presentation of 1)-3) are given in (1a), (1b), and (1c), respectively. The left side of the arrow of (1a) is deliberately kept empty. This empty place represents normal cells.

$$
\begin{gathered}
\stackrel{\mathrm{A}}{\rightarrow} \mathrm{X} \\
\mathrm{X} \stackrel{\lambda}{\rightarrow} 2 \mathrm{X} \\
\mathrm{E}_{0}+\mathrm{X} \stackrel{\mathrm{k} 1}{\rightarrow} \mathrm{E}_{1} \stackrel{\mathrm{k} 2}{\rightarrow} \mathrm{E}_{0}+\mathrm{P} .
\end{gathered}
$$

$\mathrm{X}$ denotes the population of the tumor cells. The rate of transformation from normal to neoplastic cells is proportional to a constant A. $E_{1}$ is the population of effector cells (T-lymphocytes, macrophages, natural killer cells etc. [29]) having recognized and bound a target cell (tumor cell). $\mathrm{E}_{0}$ is the population of the free effector cells, i.e., those T-lymphocytes, macrophages, natural killer cells etc., which have not yet identified and attacked a target cell. $\lambda$ is a replication rate constant. $\mathrm{k}_{1}, \mathrm{k}_{2}$ are rate constants. The first two steps represent phenomenologically two ways in which the $\mathrm{X}$ population can grow: either by transformation of the normal cells into the neoplastic ones [step (1a)] or by cellular replication [step (1b)]. In step (1c), $\mathrm{E}_{0}$ free effector cells bind (recognize and attack) $\mathrm{X}$ target cells depending upon the rate constant $\mathrm{k}_{1}$ to produce $\mathrm{E}_{1}$ number of bound effector cells. From $\mathrm{E}_{1}$ number of bound effector cells depending on another rate constant we ultimately get the number $\mathrm{E}_{0}+\mathrm{P}$, where $\mathrm{P}$ is the number of tumor cells destroyed in the operation, which will not replicate any more. We assume that $E_{t}=E_{0}+E_{1}$ is constant. Within this framework the dynamics of the growth in time of the target population $\mathrm{X}$ is given by [29], [31]

$$
\begin{aligned}
\mathrm{dX} / \mathrm{dt}=(\mathrm{N}-\mathrm{X})\{\mathrm{A}+(\lambda / \mathrm{N}) \mathrm{X}\} & \\
& -\mathrm{k}_{1} \mathrm{E}_{\mathrm{t}} \mathrm{X} /\left\{1+\left(\mathrm{k}_{1} / \mathrm{k}_{2}\right) \mathrm{X}\right\}
\end{aligned}
$$

where $\mathrm{N}$ is the maximum number of target cells which can be packed in a given volume element. Let us notice that (4.1) does not refer to the total population of these cells in the entire tumor. The problem of main interest in this model is the local transition mechanism (involving the local interactions of target and effector cells) between tissual states of different nature and not the process of growth of the tumor as a whole. Thus, the factor $(\mathrm{N}-\mathrm{X})$ expresses simply the existence of an upper limit $\mathrm{N}$ for $\mathrm{X}$ in the volume element under consideration. For the sake of convenient dynamical system theoretic analysis (4.1) can be reformulated in the following simple form:

$$
\mathrm{dm} / \mathrm{d} t=\mathrm{v}+\mathrm{m}(1-\mathrm{um})-\mathrm{r}\{\mathrm{m} /(1+\mathrm{m})\}
$$

where $\mathrm{m}=\left(\mathrm{k}_{1} / \mathrm{k}_{2}\right) \mathrm{X}, \mathrm{v}=\mathrm{k}_{1} \mathrm{AN} / \mathrm{k}_{2}(\lambda-\mathrm{A}), \mathrm{r}=\mathrm{k}_{1} \mathrm{E}_{\mathrm{t}} /(\lambda-$ $\mathrm{A}), \mathrm{u}=\mathrm{k}_{2} \lambda / \mathrm{k}_{1}(\lambda-\mathrm{A}) \mathrm{N}$, and $t=(\lambda-\mathrm{A}) \mathrm{t}$. $\mathrm{u}$ remains fixed and its value is taken as 0.1 .

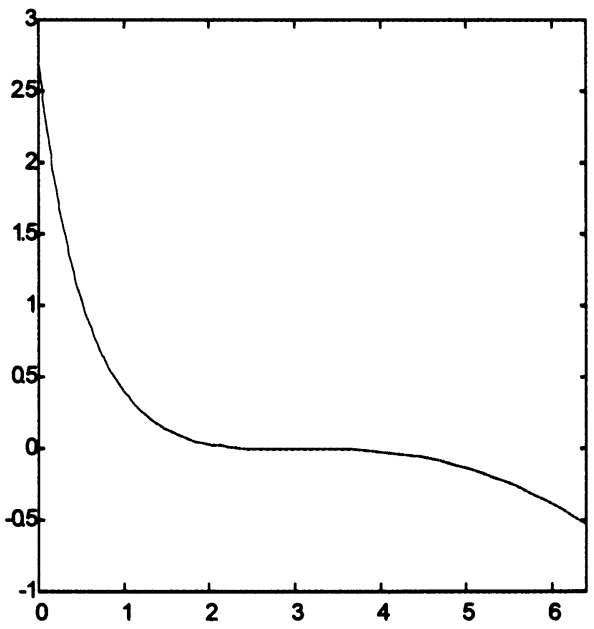

Fig. 2. Plot of $\mathrm{dm} / \mathrm{d} t$ (y-axis) against $\mathrm{m}$ (x-axis) with critical $\mathrm{r}$ and $\mathrm{v}$, i.e., $\mathrm{r}=6.4$ and $\mathrm{v}=2.7$.
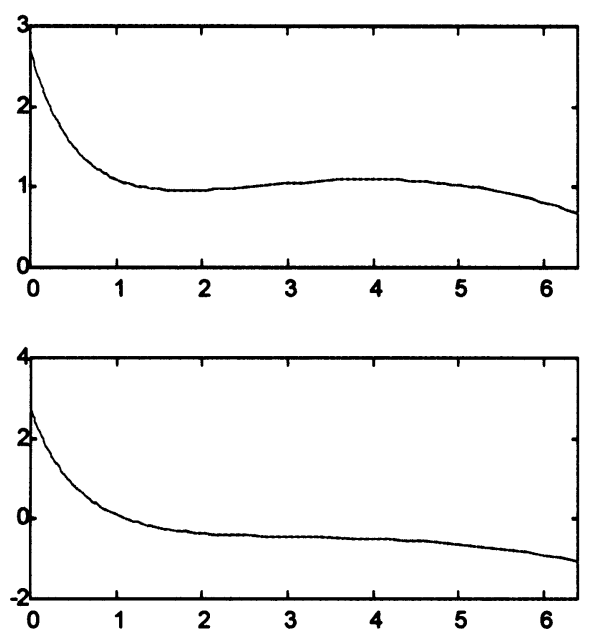

Fig. 3. Plot of $\mathrm{dm} / \mathrm{d} t$ (y-axis) against $\mathrm{m}$ ( $\mathrm{x}$-axis) for $\mathrm{r}=5$ (above) and $\mathrm{r}=7$ (below). Note that for $\mathrm{r}=5 \mathrm{dm} / \mathrm{d} t \neq 0$ when $\mathrm{m} \in[0,6]$. But for $\mathrm{r}=$ $7 \mathrm{dm} / \mathrm{d} t$ becomes 0 once for $\mathrm{m} \in[0,6]$.

\section{B. Dynamical System Theoretic Analysis}

A critical point (that is where $\mathrm{dm} / \mathrm{d} t$ vanishes) of (4.2) is given by $\mathrm{v}=(1-\mathrm{u})^{3} / 27 \mathrm{u}^{2}, \mathrm{r}=(1+2 \mathrm{u})^{3} / 27 \mathrm{u}^{2}$, and $\mathrm{m}=$ $(1-\mathrm{u}) / 3 \mathrm{u}$. Putting $\mathrm{v}=2.7$ and $\mathrm{r}=6.4$ (obtained by putting $\mathrm{u}=0.1$ in the expressions of $\mathrm{v}$ and $\mathrm{r}$ respectively) at (4.2), we have the following graph of $(\mathrm{m}, \mathrm{dm} / \mathrm{d} t)$, which shows the remarkable feature of a cusp catastrophe with an unstable saddle critical point at $\mathrm{m}=3$. For $m>3, \mathrm{dm} / \mathrm{d} t$ becomes negative, that is, the density of the malignant cells decreases with time.

In (4.2), $\mathrm{r}$ is the most important quantity from clinical and therapeutic point of view. In fact a therapy of malignancy has been suggested in [25] by an induced statistical fluctuation on $\mathrm{r}$ beyond certain threshold limit. Comparing Fig. 2 with Fig. 3, it becomes evident that 6.4 is a threshold value for $r$ (for $u=0.1$ ). If $\mathrm{r}$ is much smaller than 6.4 , the value of $\mathrm{dm} / \mathrm{d} t$ will not become negative for $0 \leq \mathrm{m} \leq 6$ (the admissible range of $\mathrm{m}$ [25]), and therefore $\mathrm{m}$, which is proportional to the malignant cell density, will not start to decrease. So to become successful any therapy must strive to fluctuate the value of $r$ beyond 6.4. Only then 
$\mathrm{dm} / \mathrm{d} t$ will become negative and $\mathrm{m}$ will start decreasing, provided of course u remains 0.1 . This way the increased fluctuation of $r$ can destabilize a tumor according to the Glansdorff-Prigogine theorem [28], which has been therapeutically utilized in [25].

Assuming that at $\mathrm{t}=0, \mathrm{~m}=0$ (when (4.2) models the growth of tumor cells then at $t=0$, we take $m=0$, but latter when from a therapeutic point of view the same equation will be used to model the diminishing of $m$ due to the therapy we shall take $\mathrm{m}=$ a high positive value at $\mathrm{t}=0)(4.2)$ has solutions of the form

$$
\exp (t)=(1-\mathrm{m} / \alpha)^{\mathrm{k}}(1-\mathrm{m} / \beta)^{l}(1-\mathrm{m} / \nu)^{\mathrm{n}}
$$

where $\alpha, \beta, \nu$ are the roots of $\mathrm{v}+\mathrm{m}(1-\mathrm{um})-\mathrm{r}\{\mathrm{m} /(1+\mathrm{m})\}=$ $-\mathrm{um}^{3}+(1-\mathrm{u}) \mathrm{m}^{2}+(\mathrm{v}-\mathrm{r}+1) \mathrm{m}+\mathrm{v}=0$, and

$$
\begin{aligned}
& \mathrm{k}=\frac{\left|\begin{array}{ccc}
0 & 1 & 1 \\
-1 & \nu+\alpha & \alpha+\beta \\
1 & \nu \alpha & \alpha \beta
\end{array}\right|}{\Delta} \\
& \Delta=\left|\begin{array}{ccc}
1 & 1 & 1 \\
\beta+\nu & \nu+\alpha & \alpha+\beta \\
\beta \nu & \nu \alpha & \alpha \beta
\end{array}\right| \\
& 1=\frac{\left|\begin{array}{ccc}
1 & 0 & 1 \\
\beta+\nu & -1 & \alpha+\beta \\
\beta \nu & 1 & \alpha \beta
\end{array}\right|}{\Delta}, \mathrm{n}=\frac{\left|\begin{array}{ccc}
1 & 1 & 0 \\
\beta+\nu & \nu+\alpha & -1 \\
\beta \nu & \nu \alpha & 1
\end{array}\right|}{\Delta} .
\end{aligned}
$$

Each of $\alpha, \beta$, and $\nu$ is a function of $\mathrm{u}, \mathrm{v}$, and $\mathrm{r}$. To know the exact form of these functions any book of theory of equations, like [32], may be consulted.

For $\mathrm{u} \ll 1$ and $\mathrm{v} \ll 1$, a bifurcation occurs in the phase space of (4.2). The values of $m$ lies on two different branches, one corresponds to low values of neoplastic cell population (microcancer focus) and the other branch corresponds to higher values of the same (macro-cancer focus). In [25] and [29] studies have been made about how to shift the focus from macro-cancer to micro-cancer through externally induced statistical fluctuations on $r$ and $\lambda$. Based on this, multi-pronged therapies of tumor have been reported in [25].

A remarkable feature of (4.3) is that for $\mathrm{m}=\alpha, \beta$ or $\nu, t$ becomes minus infinite. This means that either all of $\alpha, \beta$ and $\nu$ are negative (so that $\mathrm{m}$ can never take these values) or one is negative and the rest are complex (again so that $\mathrm{m}$ can never take these values). In the latter case, since the imaginary parts of the two complex roots have opposite signs a Hopf bifurcation [33] occurs in the phase space of (4.2). Notice that Hopf bifurcation is a multidimensional phenomenon [33] and can occur only if we allow $\mathrm{m}$ to be complex (complex plane is topologically equivalent to $\mathrm{R}^{2}$ ). Though, for our practical purpose, we never allow $m$ to be complex (to be more precise values of $m$ lies in $[0$, 6] [25]) but for the dynamical system theoretic analysis, it is illuminating to allow $\mathrm{m}$ to take all possible values (for non-negative lit t only). In (4.3) for any nonnegative $t \mathrm{~m}$ has three values. For those values of $\mathrm{u}, \mathrm{v}$, and $\mathrm{r}$ for which $\mathrm{m}$ has one real and two complex values the Hopf bifurcation occurs. This means that when the discriminant [32] of $-\mathrm{um}^{3}+(1-\mathrm{u}) \mathrm{m}^{2}+(\mathrm{v}-\mathrm{r}+1) \mathrm{m}+\mathrm{v}=$ 0 (which is a function in $\mathrm{r}$ and $\mathrm{v}$, assuming that $\mathrm{u}$ is fixed) changes sign from negative to positive a Hopf bifurcation occurs. This Hopf bifurcation is responsible for the change in stability of focus from macro-cancer to micro-cancer (or vice versa) in the dynamical system represented by (4.2).

\section{Use of FDI}

Equation (4.2) has been treated as a stochastic differential equation (SDE) of Ito or Stratonovich type in [25] and [29] and effects of various fluctuations on it have been studied. Fluctuations or, in other words, stochasticity is not intrinsic to this type of fundamental equations. Rather stochasticity arises because we do not follow up all microscopic events but rather try to describe the system macroscopically. The microscopic events are taken care of by statistical approaches. In that way fluctuations mirror a lack of knowledge. This lack of knowledge is introduced, so to speak, at our will in order not to be overloaded with unessential details [34]. Various SDEs are used to model nonlinear stochastic processes [34]. Method to solve an SDE may not always be unique. Depending upon the situation one method is preferred over the others. In the SDE model of [25] and [29] the fluctuation has been introduced as white noise, but white noise is a purely mathematical construct, which though a reasonable model of the noise encountered in physics may not be a suitable description of perturbations in biological systems [35], [41].

Reformulation of stochastic differential equations by FDIs has two advantages [41]:

1) The corresponding FDI is well defined and has a solution.

2) Using FDIs allows us to define "likelihood" and thus develop a nonstochastic analog of systems with uncertain dynamics.

The concept of FDIs generalizes the notions of differential inclusion [41].

On the other hand, from a therapeutic point of view, the induced fluctuations are not objective but subjective in nature, because these are administered by human experts according to their skill. This administration is bound to differ from one expert to another or even for a particular expert at different times. So the fluctuation should not be statistical in nature. Instead the fluctuation or the lack of knowledge involved here is fuzzy in nature. Attempts for nonstochastic modeling of uncertainty in a biological system have been made in [35], [41], where a differential inclusion modeling of population dynamics has been proposed. We have already indicated the need to introduce FDI in place of SDE as the fuzzy set theory has a very long and successful history of dealing with this kind of subjective uncertainty in complex systems [10].

In this section, we shall subject (4.2) to fuzzy fluctuations. We shall induce fuzzy fluctuations on $r$ and $v$. This means that the entire fluctuating range of $r$ and $v$ will be treated as fuzzy real numbers. Introduction of fuzzy membership functions in place of probability density functions have its own advantages and may be disadvantages also. For example, introduction of fuzzy membership function is much less rigorous and computationally less complex. It may smooth out many finer detail resulting in elimination of unnecessary information. On the other hand there is no rigorous method to construct fuzzy membership 
functions. It depends on the experience and skill of the expert who is administering the therapy by induced fluctuations.

For the therapeutic purpose fluctuations in $r$ and $v$ have been considered in [25]. So an FDI formulation of (4.2) is necessary, where without loss of generality we can take the values of $r$ and $\mathrm{v}$ lying in some fuzzy real numbers following the spirit of [35], where the noise is taken to lie within some closed and bounded interval of R. Now, the question is "What type of fuzzy real numbers are the most appropriate for this purpose?"

We have already seen that for a therapeutic purpose the value of $r$ must be influenced in such a way that it can go beyond some critical value (depending on $\mathrm{u}$, which is fixed). Like in [35], we (arbitrarily) assume some range beyond which the values of $r$ cannot go. We take $r \in[1,9]$. For $r \ll 1$ the $\mathrm{X}$ population, and hence, $\mathrm{m}$ is also close to its maximum value, and the tissue is in a cancerous state. On the contrary, if $\mathrm{r} \gg 1$, the $\mathrm{m}$ population becomes vanishingly small, and the tissue tends to normality [31]. Here, the range of $r$ is consistent with [25], [29], and [31]. The best possible range of $r$ to be attained during a therapy, to reach the micro-cancer focus, should be [5.95, 7] (which is compatible with [25]). Arbitrary large values of $r$ is not desirable, for in that case, (4.2) may not represent the dynamics properly. Since we are interested in the best possible solution only (we have discussed about the general case of the other solutions at the end of this sub-section), without loss of generality, we can take $r$ to lie in the trapezoidal fuzzy number $R_{1}=\langle 1,5.95,7,9\rangle$ for the following reason. The standard definition of fuzzy real number requires that fuzzy real number $\mu: \mathrm{R} \rightarrow[0,1]$ is a membership function, where $\mathrm{R}$ is the set of (crisp) real numbers. $\mu$ satisfies the following properties [22]. The support of $\mu$ must be a closed bounded interval of R, say, [a, b]. There must be numbers $\mathrm{c}, \mathrm{d}$, such that $\mathrm{a} \leq \mathrm{c} \leq \mathrm{d} \leq \mathrm{b}$. $\mu$ must be increasing and continuous from right in $[\mathrm{a}, \mathrm{c}] . \mu$ must be decreasing and continuous from left in $[\mathrm{d}, \mathrm{b}] . \mu(\mathrm{x})=1$ for any $\mathrm{x} \in[\mathrm{c}, \mathrm{d}]$. Since we are interested in the best possible (fuzzy) solution only, we are interested in the domain of $\mu$, where it takes the value 1 , i.e., [c, d]. We are not actually bothered about the shape of $\mu$ either in $[a, c)$ or $(d$, b]. The shape of the fuzzy number on the portion [c, d] are identical in all cases. So as long as only the best possible (fuzzy) solution is required, there is absolutely no difference between a trapezoidal fuzzy number and any other arbitrary type of fuzzy number.

Determining the proper range for the values of $\mathrm{v}$ is even more challenging. If $\mathrm{v}$, which is proportional to the rate of conversion of the normal cells into the neoplastic ones, remains positive, then after stopping the therapy, there is a chance of reviving malignancy. On the other hand, if $v$ becomes 0 during the therapy, (4.2) will give abnormal results, which means that it no longer models the dynamics properly. In Fig. 4, an approximation of the solution space of (4.2) has been presented for $\mathrm{v}=2$. The behavior of the system is very abnormal and chaotic for $m<1$. If we increase $v$, this range also increases significantly (see Fig. 5), which means that we cannot predict the progress of the therapy beyond $\mathrm{m}$ is reduced to certain level (e.g., in case of Fig. 4, it is 1). This point has not been taken care of in [25] and [29]. It is to be kept in mind that $t(\mathrm{~m})$ is a smooth function and its graph cannot exactly be like Fig. 4 or 5. Here, the graphs are generated with a resolution of $\mathrm{m}$ as fine as 0.01 . Finer resolution will give

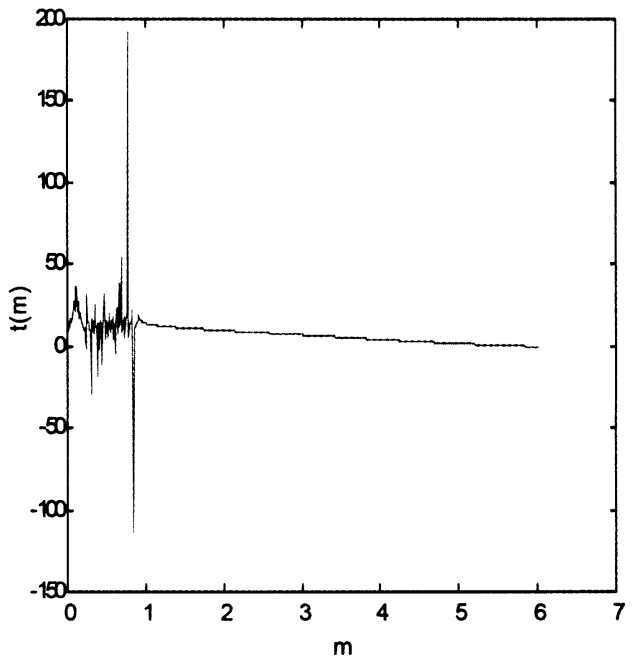

Fig. 4. Approximation of the solution space of (4.2) for $\mathrm{r}=5.95, \mathrm{v}=2$, and $\mathbf{u}=0.1$. Resolution of $\mathrm{m}$ is taken as 0.01 .

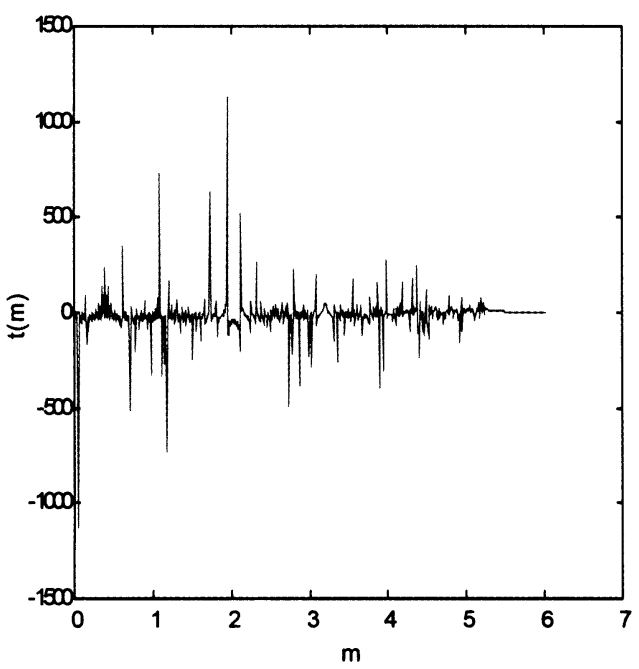

Fig. 5. Approximation of the solution space of (4.2) for $\mathrm{r}=5.95, \mathrm{v}=2.5$, and $\mathbf{u}=0.1$. Resolution of $\mathrm{m}$ is taken as 0.01 . Almost in the whole range of $\mathrm{m}$, the result is very abnormal and beyond any meaningful prediction.

more realistic figure. However, we then have the question of the size of a pixel: No resolution can be finer than that.

In reality, $v$ remains positive and due to the cytotoxicity of the T-lymphocytes tumor cell density $\mathrm{X}$, and therefore, $\mathrm{m}$ cannot grow [31]. In an abnormal situation where $m$ grows unusually, therapeutic remedy is needed. In [29], v must satisfy

$$
\mathrm{v} \ll \mathrm{r} /(1+\mathrm{u})
$$

In view of the limit of $r$ we have taken (or taken in [25], [31]) this means

$$
\mathrm{v} \leq 1 /(1+0.1) \approx 0.9
$$

For the values of $\mathrm{v}$ given by (4.5), $\mathrm{dm} / \mathrm{d} t$ is negative for $\mathrm{m}$ $\in[0,6]$ (the admissible range of $\mathrm{m}$ [25], [29]). If we take $\mathrm{r} \in$ $[5.95,7]$ then according to $(4.4) \mathrm{v} \ll 5.4$, but since our model is very sensitive to values of $\mathrm{v}$ according to this model only for $0<v \leq 2$, it is possible to devise meaningful therapy. 


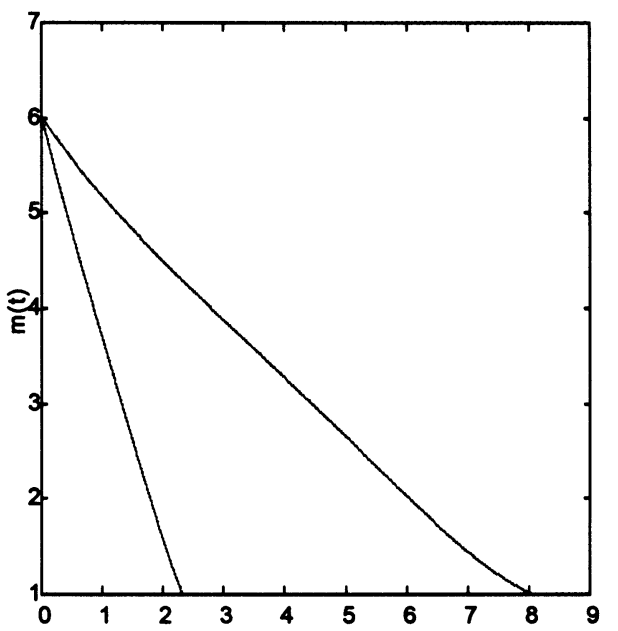

Fig. 6. Region enclosed by the curves $(t, \mathrm{~m}(t))$ for which $\mathrm{r}=7$ and $\mathrm{v}=1$ (left), $\mathrm{r}=5.95$ and $\mathrm{v}=1.75$ (right), and the $t$ axis is the region of the best possible solution (with the degree of possibility $\alpha=1$ ) of (4.6).

So we can take $\mathrm{v} \in[0.5,2]$. By introducing fuzzy membership grades in the range, we can get a trapezoidal fuzzy number $\mathrm{V}=$ $\langle 0.5,1,1.75,2\rangle$ such that $\mathrm{v} \in \mathrm{V}$.

By now, we are in a position to give an equivalent FDI formulation of (4.2).

$$
\begin{aligned}
& \mathrm{dm} / \mathrm{d} t \in {\left[\left\{-\mathrm{um}^{3}+(1-\mathrm{u}) \mathrm{m}^{2}+(\mathrm{v}-\mathrm{r}+1) \mathrm{m}+\mathrm{v}\right\} /\right.} \\
&(1+\mathrm{m})]^{\alpha} \\
& \mathrm{r} \in\left[\mathrm{R}_{1}\right]^{\alpha}, \quad \mathrm{v} \in[\mathrm{V}]^{\alpha}, \quad \mathrm{m}(0) \in[\langle 6-\delta, 6,6+\delta\rangle]^{\alpha}
\end{aligned}
$$

where $0 \leq \alpha \leq 1 .\langle 6-\delta, 6,6+\delta\rangle$ is a triangular fuzzy number, where $\delta$ is a very small positive (crisp) number. We have taken the initial condition to be the worst, i.e., at $t=0$, we have taken $\mathrm{m}(0) \in\langle 6-\delta, 6,6+\delta\rangle$, which is the highest possible value of $m$ (in [25], the maximum value of $m$ is 6). The region of best possible solution as computed according to the crystalline algorithm, described in Section II, has been shown in Fig. 6.

One important thing is to note that in Fig. 6, we have kept all our computations within the range for which $m \geq 1$. We have already seen that for $\mathrm{m}<1$ abnormal results occur. This means that for $m<1$, (4.6) no longer represents the dynamics of the system faithfully. We may have to model the dynamics for this range in a different way. This is also the case for $\mathrm{v}>2$. Designing the dynamical system for $\mathrm{m}<1$ or $\mathrm{v}>2$ is likely to pose great challenge to the future researchers.

The best possible solution region indicates the desired effects of the therapy on the patient. Due to the therapy, the possibility of $\mathrm{m}$ coming down from 6 to 1 in $t=2.3$ to 8 is very high (the degree of possibility or the value of fuzzy membership of such an happening is 1 ). Remember that $\mathrm{m}$ is the normalized (and not actual) tumor cell density, and $t$ is the normalized time. To estimate the actual value of the tumor cell density and actual value of time we must know the normalization factor. Let us remember that $\mathrm{m}=\left(\mathrm{k}_{1} / \mathrm{k}_{2}\right) \mathrm{X}$ and $t=(\lambda-\mathrm{A}) \mathrm{t}$, where $\mathrm{X}$ is the actual tumor cell density and $\mathrm{t}$ is the actual time.
For $\alpha<1$ in (4.6), we get a solution that represents the system behavior with degree of possibility $\alpha$ or more. Obviously, the solution representing the best possible system behavior is included within this solution space (or the fuzzy set of solutions, as shown in Fig. 1), as for example, for $\alpha<1$ in (4.6), the admissible range of $t$ in Fig. 6 will be a larger closed bounded interval containing $[2.3,8]$ as a proper subset. In general, for $\alpha<1$, the solution of FDI(s) tends to converge less and diverge more. Let us explain this point. The fuzzy trajectory (also known as fuzzy attainability set mapping or fuzzy reachable set mapping in the fuzzy dynamical system theory parlance), which is obtained as the fuzzy set of crisp trajectories (solutions) of the corresponding FDI(s), at any given time will give a fuzzy subset of the solution space (called fuzzy attainability set (FAS) or fuzzy reachable set). The diameter of the FAS at any given time will generally increase if $\alpha<1$ (the FAS for $\alpha=1$ is a proper subset of the FAS for $\alpha<1$ ). This makes the fuzzy trajectory obtained as the solution of the system of FDI(s) inconvenient or even completely unsuitable for the dynamical system theoretic analysis. This is exactly the reason why FDI's are preferred over fuzzy differential equations in modeling and simulation (this point has been very nicely elaborated in [39] and [40]). In FDI modeling, we have the choice to take the solution representing the best possible system behavior for the highest value of $\alpha$, which often turns out to be 1 . Once this restriction is imposed the trajectory becomes more "crisp like" (but not always crisp exactly, particularly when not all the fuzzy quantities are triangular fuzzy numbers). Crisp like trajectories are more convenient for dynamical system theoretic analysis. In the particular case of (4.6), it is obvious from the simulation that for $\alpha<1$, there will not be any qualitative change in the solution (Only [2.3, 8] will be replaced by a bigger interval properly containing this. This has been clearly demonstrated in Fig. 1 for the example treated in Section II.) We practically get the same thing by keeping $\alpha=1$. In some cases, considering the solutions for $\alpha<1$ does radically alter the whole situation. We shall come to this point in Section V.

\section{Therapy}

Any therapy devised according to the dynamics given by (4.6) must presume that 1 ) the value of $v$ will never exceed 2, and 2) after reducing $m$ to approximately 1 , the entire course of the treatment may have to be changed. This has not been taken care of in [25]. Our basic principle of therapy remains the same as in [25], where the target was to shift from the macro-cancer focus to the micro-cancer focus. Here however we shall continue this act of shifting only until $m$ comes near to 1 . For $m<1$, we cannot predict the effectiveness of the therapies under the present dynamical system modeling. At present a trial and error heuristic method is perhaps the only way out. A fluctuation on $r$ is to be induced in order it to reach the value 6.4 or even surpass it (assuming $u=0.1$ ). To fluctuate $r$, the following therapies have been suggested, which are broadly subdivided into two categories [25].

1) Multi-step therapy or multiplicative therapy: Here, we can use perturbation in the form of increasing arterial $\mathrm{pO}_{2}$ to $90 \mathrm{mmHg}$, decreasing venus $\mathrm{pO}_{2}$ to $20 \mathrm{mmHg}$, 
using hyperthermia up to $105^{\circ} \mathrm{F}$, hyperglycaemia up to $600 \mathrm{mg} \%$ blood glucose level, which also produce $\mathrm{pH}$ perturbations (up to 6.5 from the normal tissue level of 7.8). Owing to variation of oxygenation, there is a perturbation in the oxygen index of the blood $\eta$ as $18.5<\eta<$ 42.

2) Multiplex therapy: Here, one uses the combined perturbation of glucose level, temperature, anti-tumor drugs, radiation, thermolabilizer, and radiosensitizer. Concentrated glucose solution is administered through superior vena caval catheter and the following perturbations are induced:

a) Hyperglycaemia-Glucose level is varied between $400 \mathrm{mg} / 100 \mathrm{ml}$ blood and normal level of 100 $\mathrm{mg} / 100 \mathrm{ml}$ blood.

b) Hyperthermia-Temperature variation used is from $98^{\circ} \mathrm{F}$ to $103^{\circ} \mathrm{F}$, using high-frequency inductive heating.

c) Oxygenation-This is administered through pulmonary ventilation.

Success has been reported under both the above categories of therapies in [25]. If we examine the region of the phase space of (4.6) corresponding to the best possible solution (see Fig. 6), we observe that under the therapies $m$ reduces from 6 to 1 quite smoothly. This means that the cancer cell population $\mathrm{X}$ also reduces accordingly. The corresponding values of $t$ lies in the range of 2.3 to 8 (remember that $t$ is not time but proportional to time). Within this best possible solution region of the FDI relation (4.6), the response to the therapies is quite reasonable and predictable, just as in case of the conventional SDE modeling.

\section{Summary AND CONCLUSION}

We started this paper with an investigation into the role of FDIs in the atmospheric and bio-medical cybernetics. The mathematical theory of FDS in general and the FDI in particular have so far found few applications [9], [37], [41] despite their immense potentiality to model complex systems. We have discussed in detail the complexity dealing aspects of the FDS in general and the FDI in particular in [10].

So far, only the first-order and first-degree FDIs have been considered in literature. However, in Section II, we have applied Crystalline Algorithm to solve the 1-D second-order FDIs. Later in Section IV, we have solved FDIs modeling the evolution of tumor in human body.

In Section III, the notion of cybernetics has been extended to the atmospheric sciences, where we have reviewed the FDI modeling of a climatic disturbance leading to cyclogenesis as described in detail in [9], which is the first known application of the fuzzy set theory based techniques in the atmospheric science (it is interesting to note that the fuzzy set theory-based techniques were applied to the earth science in 1986 [56]). One of the present authors has recently presented a model of turbulence in terms of FDIs [55], [60]. This modeling technique can be extended to model atmospheric turbulence. The emphasis here will be on presenting an FDI modeling of the genesis of a storm from atmospheric turbulence. Unlike the FDIs treated in the current paper, it will be shown that in the modeling of the genesis of a storm from the atmospheric turbulence the solutions of FDIs for $\alpha<1$ play very important roles. It should be appreciated that treating the solutions of a set of FDIs for $\alpha=1$ and $\alpha \neq 1$ are two different paradigms ([39] and [40] may be consulted to have a feel of it).

In Section IV, a dynamical system theoretic study of the evolution of tumor in a human body has been made. Here, we have followed the precedence set in [25], [29], and [31], but we have gone even further. We have shown that an FDS (FDI to be more precise) modeling of a biological system is no less effective than a modeling in terms of the stochastic differential equations. Again, this is the maiden effort to introduce FDI relations into the realm of biomedical cybernetics. We have undertaken the dynamical system theoretic analysis of the model of Lefever and Horsthemke [29] in a new direction and discovered the range within which the model should work. Based on these limitations, therapeutic suggestions have been made. This shows that a more pragmatic therapeutic planning needs to be introduced in treating malignancy, as suggested in [25]. Our study opens up the need for research on a new dynamical system to model the evolution of tumor in human tissues when either 1) the neoplastic cell density $\mathrm{X}$ and hence, $\mathrm{m}$ becomes very low $(\mathrm{m}<1)$, or 2) the conversion rate from normal cells to the neoplastic ones becomes very high (for which $\mathrm{v}>2$ ) or both. The authors believe that these two are the most important open problems suggested by the present work.

Since both the systems discussed in Sections III and IV are uncertain systems, we have been able to model them using FDIs. In Section IV, we have mentioned where ever the system behaves chaotically (remember that in this paper, for simplicity, we have interpreted chaotic as unpredictable). In Fig. 4, the system behaves chaotically for $\mathrm{m}[0,1]$, and in Fig. 5, the system behaves chaotically in the entire range of $\mathrm{m}$. It will be interesting to determine the fractal dimension of the graphs of Figs. 4 and 5 in the euclidean plane. By fractal dimension, we mean the Hausdorff-Besicovitch dimension [57]. The problem will be a little easier if we take the box counting dimension [57] as the fractal dimension. If the fractal dimension is a number between 1 and 2 , that is a good indication that the system may behave chaotically.

\section{ACKNOWLEDGMENT}

The authors would like to acknowledge all the five anonymous referees and the Associate Editor for constructive reviewing and helpful suggestions. They also acknowledge Dr. V. Krivan for promptly sending one of his papers [41]. Reference [44] has turned out to be a useful reference during simulation works.

\section{REFERENCES}

[1] W. R. Ashby, An Introduction to Cybernetics. London, U.K.: Methuen, 1976.

[2] E. Hullermeier, "An approach to modeling and simulation of uncertain dynamical systems," Int. J. Uncertainty, Fuzziness, Knowledge-Based Syst., vol. 5, pp. 117-137, 1997.

[3] N. Wiener, Cybernetics: Or Control and Communication in the Animal and the Machine. New York: Wiley, 1949.

[4] S. S. L. Chang and L. A. Zadeh, "On fuzzy mappings and control," IEEE Trans. Syst. Man, Cybern., vol. SMC-2, pp. 30-34, 1972. 
[5] P. E. Kloeden, "Fuzzy dynamical systems," Fuzzy Sets Syst., vol. 7, pp. 275-296, 1982

[6] M. de Glas, "Theory of fuzzy systems," Fuzzy Sets Syst., vol. 10, pp. 65-77, 1983.

[7] O. Kaleva, "Fuzzy differential equations," Fuzzy Sets Syst., vol. 24, pp. 301-317, 1987

[8] S. Seikkala, "On the fuzzy initial value problem," Fuzzy Sets Syst., vol. 24, pp. 319-330, 1987.

[9] K. K. Majumdar, "A fuzzy dynamical system modeling of a disturbance leading to cyclogenesis," J. Intell. Fuzzy Syst., vol. 13, pp. 7-15, 2003.

[10] D. D. Majumder and K. K. Majumdar, "Complexity analysis, uncertainty management, and fuzzy dynamical systems: A cybernetic approach with some case studies," Kybernetes, to be published.

[11] L. A. Zadeh, "Outline of a new approach to the analysis of complex systems and decision processes," IEEE Trans. Syst. Man, Cybern., vol. SMC-3, pp. 28-44, 1973

[12] E. Hullermeier, "Numerical methods for fuzzy initial value problems," Int. J. Uncertainty, Fuzziness, Knowledge-Based Syst., vol. 7, pp. 1-23, 1999.

[13] H. T. Nguyen, "A note on the extension principle for fuzzy sets," J. Math. Anal. Appl., vol. 64, pp. 369-380, 1978.

[14] V. F. Dvorak, "Tropical cyclone intensity analysis using satellite data," Satellite Applications Lab., Washington, DC, NOAA Tech. Rep. NESDIS 11, 1984.

[15] K. A. Emanuel, "Toward a general theory of hurricanes," Amer. Sci., vol. 76, pp. 370-379, 1988

[16] J. C. Mandal, "A semi-implicit multiple-nested grid model for simulation of flow in a tropical storm," Mausam, vol. 47, pp. 1-20, 1996.

[17] K. Ooyama, "Numerical simulation of life-cycle of tropical cyclones," J. Atmos. Sci., vol. 26, pp. 3-40, 1969.

[18] R. Rotuno and K. A. Emanuel, "An air-sea interaction theory for tropical cyclones. Part II: Evolutionary study using a nonhydrostatic axisymmetric numerical model," J. Atmos. Sci., vol. 44, pp. 542-561.

[19] P. Bouthemy and A. Benveniste, "Modeling of atmospheric disturbances in meteorological pictures," IEEE Trans. Patt. Anal. Machine Intell., vol. PAMI-6, pp. 587-600, 1984.

[20] R. A. Anthes, "Tropical cyclones: Their evolution, structures, and effects," Amer. Meteorol. Soc., Mono. no. 41, 1982.

[21] R. A. Brown, Fluid Mechanics of the Atmosphere. San Diego, CA: Academic, 1991.

[22] G. J. Klir and B. Yuan, Fuzzy Sets and Fuzzy Logic: Theory and Applications. New Delhi, India: Prentice-Hall, 1997.

[23] A. Kandel, Y. Luo, and Y.-Q. Zhang, "Stability analysis of fuzzy control systems," Fuzzy Sets Syst., vol. 105, pp. 33-48, 1999.

[24] D. D. Majumder, "Cybernetics and general systems-A unitary science," Kybernetes, vol. 8, pp. 7-15, 1979.

[25] D. D. Majumder and P. K. Roy, "Cancer self-remission and tumor instability-A cybernetic analysis: Toward a fresh paradigm for cancer treatment," Kybernetes, vol. 29, pp. 896-927, 2000.

[26] P. Roy, D. D. Majumder, and J. Biswas, "Spontaneous cancer regression: Implications for fluctuation," Ind. J. Phys., vol. 73-B, pp. 777-883, 1999.

[27] W. Horsthemke and R. Lefever, Noise-Induced Transitions: Applications to Physics, Chemistry, and Biology, Berlin: Springer, 1984.

[28] P. Glansdorff and I. Prigogine, Thermodynamic Theory of Stability, Structure and Fluctuation. London, U.K.: Wiley, 1971.

[29] R. Lefever and W. Horsthemke, "Bistability in fluctuating environments. Implications in tumor immunology," Bull. Math. Biol., vol. 41, pp. 469-490, 1979.

[30] R. Lefever and R. P. Garay, "A mathematical model of the immune surveillance against cancer," in Theoretical Immunology, G. I. Bell, A. S. Perelson, and G. H. Pimbley, Eds. New York: Marcel Dekker, 1978.

[31] R. P. Garey and R. Lefever, "A kinetic approach to the immunology of cancer: Stationary state properties of effector-target cell reactions," $J$. Theoretical Biol., vol. 73, pp. 417-438, 1978.

[32] L. E. Dickson, New First Course in the Theory of Equations. New York: Wiley, 1947.

[33] T. Kapitaniak and S. R. Bishop, The Illustrated Dictionary of Nonlinear Dynamics and Chaos. Chichester, U.K.: Wiley, 1999.

[34] H. Haken, "Transition phenomena in nonlinear systems," in Stochastic Nonlinear Systems in Physics, Chemistry, and Biology, L. Arnold and R. Lefever, Eds, Berlin, Germany: Springer-Verlag, 1981.

[35] V. Krivan and G. Colombo, "A nonstochastic approach for modeling uncertainty in population dynamics," Bull. Math. Biol., vol. 60, pp. 721-751, 1998

[36] J.-P. Aubin, "Fuzzy differential inclusions," Probl. Cont. Inform. Theory, vol. 19, pp. 55-57, 1990.
[37] K. K. Majumdar, "A mathematical model of the nascent cyclone," IEEE Int. Geosci. Remote Sensing, vol. 41, pp. 1118-1122, 2003.

[38] L. V. Bertalanffy, General System Theory. New York: George Brazillier, 1968.

[39] P. Diamond, "Time-dependent differential inclusions, cocycle attractors and fuzzy differential equations," IEEE Trans. Fuzzy Syst., vol. 7, pp. 734-740, Dec. 1999.

[40] - "Stability and periodicity in fuzzy differential equations," IEEE Trans. Fuzzy Syst., vol. 8, pp. 583-590, Oct. 2000

[41] P. Antonelli and V. Krivan, "Fuzzy differential inclusions as substitutes for stochastic differential equations in population biology," in VolterraHamilton Models in the Ecology and Evolution of Colonial Organizms. ser. Series in Mathematical Biology and Medicine, P. L. Antonelli and R. H. Bradbury, Eds. Singapore: World Scientific, 1996, vol. 2.

[42] K.-P. Adlassnig, "Fuzzy set theory in medical diagnosis," IEEE Trans. Syst. Man, Cybern, vol. SMC-16, pp. 260-265, 1986.

[43] M. L. Tsetlin, Automaton Theory and Modeling of Biological Systems. New York: Academic, 1973

[44] L. V. Fausett, Applied Numerical Analysis Using MATLAB. Englewood Cliffs, NJ: Prentice-Hall, 1999.

[45] B. B. Mandelbrot, The Fractal Geometry of Nature. New York: W.H. Freeman, 1982

[46] A. Mees and C. Sparrow, "Some tools for analyzing chaos," Proc. IEEE, vol. 75, pp. 1058-1070, 1987.

[47] E. Ott, C. Grebogi, and J. A. York, "Controlling chaos," Phys. Rev. Lett., vol. 64, pp. 1196-1199, 1990.

[48] P. Diamond, "Chaos and fuzzy representations of dynamical systems," in Proc. 2nd Int. Conf. Fuzzy Logic Neural Networks, Iizuka, Japan, July 17-22, 1992, pp. 51-58.

[49] H.-N. Teodorescu, "Chaos in fuzzy systems and signals," in Proc. 2nd Int. Conf. Fuzzy Logic Neural Networks, Iizuka, Japan, July 17-22, 1992, pp. 21-50.

[50] P. E. Kloeden, "Chaotic iteration of fuzzy sets," Fuzzy Sets Syst., vol. 42, pp. 37-42, 1991.

[51] J. J. Buckley and Y. Hayashi, "Applications of fuzzy chaos to fuzzy simulation," Fuzzy Sets Syst., vol. 99, pp. 151-157, 1998.

[52] R. L. Devaney, An Introduction to Chaotic Dynamical Systems. Redwood City, CA: Addison-Wesley, 1989.

[53] M. F. Barnsley, Fractals Everywhere. San Diego, CA: Academic, 1988.

[54] C. A. Cabrelli, B. Forte, U. M. Molter, and E. R. Vrscay, "Iterated fuzzy sets systems: A new approach to the inverse problem for fractals and other sets," J. Math. Anal. Appl., vol. 171, pp. 79-100, 1992.

[55] K. K. Majumdar, "Some studies on uncertainty management in dynamical systems by fuzzy techniques with applications," $\mathrm{Ph}$.D. dissertation, Indian Statist. Inst., Calcutta, India, Mar. 2003. submitted.

[56] "Bulletin Section," Fuzzy Sets Syst., vol. 24, 1987.

[57] G. A. Edgar, Measure, Topology, and Fractal Geometry. New York: Springer-Verlag, 1990.

[58] A. Kandel, Y. Lou, and Y.-Q. Zhang, "Stability analysis of fuzzy control systems," Fuzzy Sets Syst., vol. 105, pp. 33-48, 1999.

[59] K. S. Ray and D. D. Majumder, "Application of circle criteria for stability analysis of linear SISO and MIMO systems associated with fuzzy logic controllers," IEEE Trans. Syst. Man, Cybern., vol. SMC-14, pp. 345-349, 1984

[60] K. K. Majumdar, "Fuzzy fractals and fuzzy turbulence," IEEE Trans. Syst., Man, Cybern. B, to be published.

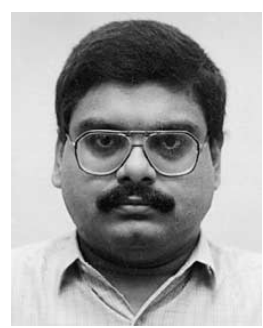

Kausik Kumar Majumdar was born in 1967. He received the B.Sc. and M.Sc. degrees in mathematics from the University of Calcutta, Calcutta, India, and Annamalai University, Tamil Nadu, India, respectively. He received the M.Tech. and Ph.D. degrees in computer science from the Indian Statistical Institute (ISI), Calcutta.

$\mathrm{He}$ is currently a Research Associate of the Council of Scientific and Industrial Research of the Government of India and working at the Electronics and Communication Sciences Unit of the ISI. His areas of interest are fuzzy and conventional dynamical systems and their applications in earth, atmospheric, and physical sciences. As part of his current CSIR Research Associateship, he is working on some aspects of modeling of tropical cyclones and the evolutionary dynamics of tumor.

Dr. Majumdar is a member of American Mathematical Society. 


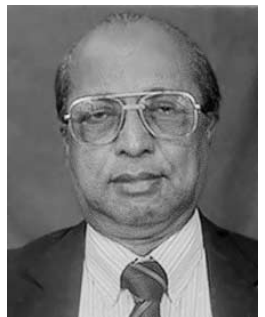

Dwijesh Dutta Majumder was born in 1932. He received the B.Sc. degree in physics from Guwahati University, Assam, India, in 1952 and the M.Sc. and $\mathrm{Ph} . \mathrm{D}$. degrees from the University of Calcutta, Calcutta, India, in 1955 and 1963, respectively.

He is currently an Emeritus Professor of the Indian Statistical Institute, Calcutta, and an Emeritus Scientist of CSIR. He is the Director-Secretary of the Institute of Cybernetics Systems and Information Technology, Calcutta. He is a member of the Board of Directors of the World Organization of Systems and $\mathrm{Cy}$ bernetics, Paris, France. He is a member of Indo-US Program on Neuroscience between the National Institute of Health, USA, and National Brain Research Centre, India. He was a visiting scientist at Carnegie Mellon University, Pittsburgh, PA; Stanford University, Stanford, CA; Oxford University, Oxford, U.K. and the University of Maryland, College Park. His current research interests include developing knowledge-based systems that incorporate his unitary formalism of cybernetics and general systems theory in integrated human-machine systems, unifying observing, observed, and the act of observing in mathematical framework of second-order cybernetics.

Dr. Majumder is a Fellow of Indian National Science Academy, the Third World Academy of Sciences, and the International Association of Pattern Recognition (India). He received the UN Fellowship from the University of Michigan, Ann Arbor, in 1964, the IETE Sir J. C. Bose Award in 1969, the International Norbert Wiener Award in 1977, the IETE R. L. Wadhwa Gold Medal in 1982, the CSI award in 1983, the Sir C. V. Raman Award in 1985 , the Prasanta Chandra Mahalanobis Award, the Gold Medal of INSA in 1993, the INSA Jawaharlal Nehru Birth Centenary Lecture Award in 1994, the IETE K. S. Krishnan Gold Medal in 1994, the IETE S. K. Mitra Memorial Award in 1998, the Frank George Research Award of the WOCS and Kybernetes in 1999, and the Rabindra Puraskar Award 2001. He is the founding president of the Indian Society of Fuzzy Mathematics and Information Processing (ISFUMIP) and governing Board Member of the International Fuzzy Systems Association (IFSA) and IAPR. He is also the Founder and President of Indian unit for Pattern Recognition and Artificial Intelligence. 